\title{
1 Optical Cellular Micromotion: A New Paradigm to Measure 2 Tumour Cells Invasion in 3D Tumour Environments
}

3 Zhaobin Guo ${ }^{1}$, Chih-Tsung Yang ${ }^{1}$, Chia-Chi Chien ${ }^{l}$, Luke A. Selth ${ }^{2,3}$, Pierre

$4 \quad$ O. Bagnaninchi ${ }^{4} *$ and Benjamin Thierry ${ }^{1} *$

$5 \quad{ }^{1}$ Future Industries Institute and ARC Centre of Excellence in Convergent Bio-Nano

6 Science and Technology, University of South Australia, Mawson Lakes, SA, Australia

$7 \quad{ }^{2}$ Dame Roma Mitchell Cancer Research Laboratories and Freemasons Foundation Centre

8 for Male Health and Wellbeing, Adelaide Medical School, The University of Adelaide,

9 SA, Australia.

$10{ }^{3}$ Flinders Health and Medical Research Institute, Flinders University, Bedford Park, SA, 11 Australia.

$12{ }^{4}$ Centre for Regenerative Medicine, University of Edinburgh, UK.

13 Benjamin Thierry, Pierre O. Bagnaninchi

14 Email: benjamin.thierry@unisa.edu.au, pierre.bagnaninchi@ed.ac.uk;

15 Author Contributions: B.T., P.B., and Z.G. designed the study. Z.G. and B.T. designed 16 the experiments, P.B. established the micromotion index calculation algorithm, Z.G. and 17 C.Y. established the on-chip vasculature and conducted the extravasation study. L.S. 18 established the prostate cancer model and conducted the animal study, Z.B. and C.C. 19 designed the micromotion measurement setup. All authors reviewed the data, Z.G. and 20 B.T. wrote the manuscript. All authors edited and provided critical feedback on the 21 manuscript.

22 Competing Interest Statement: The authors declare no conflict of interest. 
1 Classification: Biological Sciences >>Cell Biology, Physical Sciences $>>$ Applied

2 Physical Sciences

3 Keywords: micromotion, 3D cell environment, invasion, migration, digital holographic

4 microscopy (DHM)

\section{Abstract}

6 Measuring tumour cell invasiveness through three-dimensional (3D) tissues, particularly

7 at the single cell level, can provide important mechanistic understanding and assist in

8 identifying therapeutic targets of tumour invasion. However, current experimental

9 approaches, including standard in vitro invasion assays, have limited physiological

10 relevance and offer insufficient insight about the vast heterogeneity in tumour cell

11 migration through tissues. To address these issues, here we report on the concept of

12 optical cellular micromotion, where digital holographic microscopy (DHM) is used to

13 map the optical thickness fluctuations at sub-micron scale within single cells. These

14 fluctuations are driven by the dynamic movement of subcellular structures including the

15 cytoskeleton and inherently associated with the biological processes involved in cell

16 invasion within tissues. We experimentally demonstrate that the optical cellular

17 micromotion correlates with tumour cells motility and invasiveness both at the population

18 and single cell levels. In addition, the optical cellular micromotion significantly reduced

19 upon treatment with migrastatic drugs that inhibit tumour cell invasion. These results

20 demonstrate that micromotion measurements can rapidly and non-invasively determine

21 the invasive behaviour of single tumour cells within tissues, yielding a new and powerful

22 tool to assess the efficacy of approaches targeting tumour cell invasiveness.

\section{Significance Statement}

25 Tumour cells invasion through tissues is a key hallmark of malignant tumour progression

26 and its measurement is essential to unraveling biological processes and screening for new

27 approaches targeting cell motility. To address the limitations of current approaches, we

28 demonstrate that sub-micron scale mapping of the dynamic optical thickness fluctuations 
1 within single cells, referred to as optical cellular micromotion, correlates with their

2 motility in ECM mimicking gel, both at the population and single cell levels. We

3 anticipate that 3D optical micromotion measurement will provide a powerful new tool to

4 address important biological questions and screen for new approaches targeting tumour

5 cell invasiveness.

$7 \quad$ Main Text

\section{Introduction}

9 Metastasis is the main direct cause of tumour-related mortality. It is initiated by tumour 10 invasion to nearby/distant organs as a result of tumour cells breaking the basement 11 membrane barrier and migrating into adjacent tissues. Tumour cell invasion is therefore a

12 key hallmark of malignant progression. Investigation of factors that drives tumour cell

13 invasion has the potential to reveal new approaches to control early stage diseases ${ }^{1,2}$ as

14 well as to provide better understanding of the role of tumour cell phenotypes and tumour 15 microenvironment on the metastatic process ${ }^{3-6}$. While a number of conventional cell 16 invasion assays exists, they suffer from several limitations that negatively impact 17 progress in this field. For example, commonly used wound healing or Boyden chamber 18 assays are laborious and time consuming, typically requiring hours or days for data 19 acquisition. Moreover, the readout of these assays are accumulative invasion effects 20 between observation intervals, such as migration distance or coverage of wounds, which

21 fail to inform on the transient invasiveness changes in response to external factors. More

22 importantly, these assays do not recapitulate the 3D environment experienced by cancer 23 cells in intricate contact with the extracellular matrix $(\mathrm{ECM})^{7-9}$, which limits their 24 relevance for the screening of potential activators or suppressors of tumour invasion.

25 Finally, current invasion/migration assays are typically population-based, and thereby do 26 not provide insights about the role of tumour cells heterogeneity in these processes.

27 The invasion of tumour cells within tissues is inherently associated with cellular 28 structural changes, including the formation of lamellipodia, filopodia, and integrin29 mediated adhesion $^{10,11}$, in which the cytoskeleton (e.g. actin, myosin, etc.) plays a pivotal 
1 role. This led us to hypothesize that, independently of the upstream effectors of invasion,

2 the invasiveness of a specific tumour cell within its 3D microenvironment can be directly

3 correlated to the cellular micromotion. Cellular micromotion refers to the biologically-

4 driven continuous nanoscale movement of the cell membrane and intracellular structures,

5 including the cytoskeleton. ${ }^{12-15}$ Micromotion can be experimentally measured using

6 electrical impedance changes associated with nanoscale to submicron scale fluctuations

7 of the membrane of living cells on microelectrodes, as initially described by Giaever and

$8 \mathrm{Keese}^{12}$. In support of this early pioneering work, several studies have since shown that

9 cellular micromotion as measured by electrical impedance can not only identify

10 cancerous cells but also provide insight into their metastatic potential ${ }^{13,15,16}$. In addition,

11 phenotypic changes linked to invasion such as epithelial mesenchymal transition (EMT),

12 are also associated with measurable changes in cellular micromotion ${ }^{17,18}$.

13 While the monitoring of cellular micromotion therefore provides a powerful approach to 14 measuring, within a few minutes, the invasiveness of tumour cells, the current modus 15 operandi based on impendence measurements suffers from several conceptual and 16 practical limitations. The monitoring of micromotion by impedance requires the 17 formation of a cell monolayer onto the solid substrate serving as electrode. While this 18 makes for an easy experimental set-up, it presents limited physiological relevance to the 19 invasiveness of cells within the 3D environment of real tissues. In addition, it does not 20 allow for single-cell measurements and consequently provides no insights into the 21 inherent heterogeneity of the invasion process.

22 To validate our hypothesis that cell invasiveness has a significant correlation with cell 23 micromotion, and to overcome the shortcomings of impedance-based measurements of 24 the micromotion, we endeavoured to measure the micromotion of individual cells within 25 a 3D environment by monitoring and processing the spatiotemporal fluctuations of the 26 optical thickness. To this end, we utilized digital holographic microscopy (DHM) to 27 record the instantaneous optical thickness fluctuations of single cells embedded within 28 fibrin gels and developed a dedicated algorithm to obtain a 3D micromotion index from 29 these measurements. To demonstrate the link between the $3 \mathrm{D}$ micromotion index and 
1 invasiveness, the breast cancer cell line MDA-MB-231 was treated with epidermal 2 growth factor (EGF) to induce a more invasive phenotype. The micromotion indexes of

3 the treated cells suspended in a fibrin gel were significantly increased in comparison to

4 the untreated ones. Further, we demonstrate that the 3D micromotion index is correlated

5 at the single cell level to their motility within the fibrin gel measured as the mean

6 migration speed over $12 \mathrm{~h}$. To further validate our hypothesis, we showed that cells in

7 more invasive mesenchymal phenotypes also displayed significantly increased 3D

8 micromotion indexes. A direct correlation was also measured at the single cell level

9 between vimentin expression and micromotion for A549 cells. In addition, the

10 micromotion of miR-194 overexpressing PC-3 cells was measured and compared to that

11 of normal PC-3 cells. We have previously shown that miR-194 is a driver of prostate

12 cancer invasiveness and stable overexpression enhanced metastasis of intravenous and

13 intraprostatic tumor xenografts ${ }^{19}$. MiR-194 PC-3 cells were also found to have

14 significantly higher extravasation rates in a microfluidic vasculogenesis model and

15 yielded significantly higher micromotion than that of non-transfected cells. Finally, PC-3

16 cells treated with cellular motility targeting (migrastatic) compounds that have been

17 shown to inhibit prostate cancer cells invasion and metastasis ${ }^{20,21}$ yielded significantly

18 reduced micromotion, and the dynamic of micromotion alteration is in good agreement

19 with the effectiveness of compounds in invasion inhibition. Altogether, this data validates

20 the concept that a 3D micromotion index obtained from dynamic phase measurements

21 provides rapid and simple measurement of tumour cells invasiveness in the 3D

22 environment at the single cell level, and shows the feasibility of using optical 3D cellular

23 micromotion to investigate the effectiveness of novel migrastatic approaches. The

24 application of this new paradigm could accelerate the screening for novel therapies as

25 well as assist in addressing important biological questions about the metastatic process.

\section{Results}

27 Probing and quantifying optical cellular micromotion in 3D environments. We first

28 endeavoured to demonstrate the feasibility of measuring the dynamic optical thickness

29 (OT) fluctuations of cells within a 3D environment mimicking that of tumour tissues. We 
1 selected fibrin gel as the model 3D extracellular matrix due to its inherent high

2 bioactivity and suitability for studies related to cellular adhesion and degradation

3 processes. Fibrin gel promotes typical invasive behaviours, for instance

4 microenvironment remodelling and migration and is commonly used in advanced tumour

5 models, both in vivo and in vitro ${ }^{22,23}$. MDA-MB-231 breast cancer cells were embedded

6 in $5 \mathrm{mg} / \mathrm{mL}$ of fibrin gel and loaded in a custom-made microfabricated device, in which

7 both cells and gels are sandwiched between two coverslips. This configuration provides a

8 homogeneous optical background required for high precision phase imaging with DHM.

9 Holographic movies of single cells in the fibrin gels were captured and converted to quantitative phase images (Fig. 1A and SI movie 1), from which the phase shifts were extracted and segmented from background (Fig. 1B-1D). DHM requires only low

12 intensity illumination, and it is therefore possible to perform imaging continuously at 13 high capture frequencies and/or repeatedly. In our experimental set-up, high frequency 14 acquisition enabled the capture of the fluctuations of the OT for whole cells (Movie S1).

15 Micromotion indexes have been previously obtained by calculating the mean square of 16 the stochastic signal fluctuations associated with the presence of live cells on the solid 17 substrates ${ }^{24-26}$. In the proposed optical micromotion paradigm, one can either consider 18 the OT fluctuations for an entire cell (e.g. averaging the fluctuation of all the pixels 19 comprised within the cell) or the fluctuation of every pixel within a single cell. To 20 illustrate the difference between the two approaches, we randomly selected 3 pixels 21 inside a cell and plotted the fluctuations of the associated OT over time. We also plotted 22 the mean OT calculated for the same whole cell (Fig. S1). From these measurements, it is 23 clear that the process of averaging results in significant dampening of the OT 24 fluctuations. This is not surprising as over the short period of time of these 25 measurements, the overall OT of a cell is relatively constant. On the other hand, 26 biological processes result in significant redistribution of the dry mass of 'optically thick' 27 intracellular structures, which can be detected when monitoring single pixels. Dry mass 28 refers to the mass of all the substances within the cells (e.g. protein, nucleic acid, etc.) 29 other than water. We therefore quantified the dynamic fluctuation of the OT for every 
1 pixel inside each cell (Eq. 2), and then averaged these values over the whole cell (Eq. 3)

2 to obtain the single cell MI. The entire measurement process is illustrated in Fig. 1E-J.

3 Measured 3D optical cellular micromotion are mainly associated with live cell

4 behaviours. To confirm that the calculated MI is associated with biological processes,

5 we compared values obtained for live cells with those obtained for fixed cells, both in the

$63 \mathrm{D}$ and 2D (cells plated on a coverslip) environment. As shown in Fig. 2A and B,

7 significantly lower fluctuations were observed for fixed cells both in $2 \mathrm{D}$ and $3 \mathrm{D}$. The

8 calculated MI indexes of 30 individual cells are presented in Fig. 2C. The MIs of live

9 MDA-MB-231 cells ranged from 0.07 to 0.27 (average of 0.165 ) in the 3D environment

10 while the MIs of fixed cells ranged from 0.02 to 0.04 (average of 0.032). Similarly, the

11 averaged MI for live cells in 2D was 0.07 while the average MI for fixed cells was 0.018 .

12 This data confirm that the measured dynamic fluctuations are mainly associated with live

13 cell rather than to instrumental noise or physical perturbations. The MI was also

14 calculated at $6 \mathrm{~h}$ or $24 \mathrm{~h}$ after being seeded into the $3 \mathrm{D}$ fibrin gel, and no significant

15 differences were found neither for the MDA-MB-231 nor MCF-7 cells (Table. S1).

16 Measured 3D optical cellular micromotion are significantly higher than that in 2D.

17 Our initial experiments with the MDA-MB-231 model also allowed us to directly 18 compare the cellular micromotion in 2D and 3D environments (Fig. 2A and B, Movie S1

19 and S2). The mean MI of the MDA-MB-231 cells in 3D fibrin gel was significantly

20 higher than that of cells plated on a cover-slip (2D) $(0.165 \pm 0.045$ vs $0.069 \pm 0.021, \mathrm{p}<$

21 0.001). Meanwhile, the minor MIs difference between fixed cells in 3D and 2D (0.032 \pm

220.004 vs $0.018 \pm 0.004)$ indicates that the significant differences observed for the live

23 cells between the 2D and 3D environments indeed originates from biological phenomena

24 instead of experimental or technical discrepancies. While the reason for the difference of

25 MIs between in 2D and 3D is not clear, it likely originates in the increased rigidity of

26 cells adhered to aberrantly hard substrates which limits cell membrane and intracellular

27 motility. On the other hand, it is also likely that cells inherently display higher

28 mechanobiological activities or different modality of invasion when in a 3D environment

29 mimicking that of real tissues ${ }^{27,28}$. 
1 Micromotion and invasiveness in fibrin gel are correlated at the single cell level. We

2 next investigated whether micromotion measurements could be correlated to the

3 invasiveness of cells within a 3D environment. To modulate the invasive capability of

4 MDA-MB-231 cells, we treated cells with the epidermal growth factor (EGF), which

5 increases invasiveness in vitro and in vivo ${ }^{29,30}$. The MDA-MB-231 cells were dispersed

6 within a 3D fibrin gel supplemented or not with $100 \mathrm{ng} / \mathrm{mL}$ EGF. The micromotion

7 indexes of the MDA-MB-231 cells was then measured from the DHM as described above

8 at $6 \mathrm{~h}$ after seeding (Fig. 3A). Following DHM imaging, these cells were individually

9 tracked to establish their migration paths in the following $12 \mathrm{~h}$, taking an image every 10

$10 \mathrm{~min}$. Herein we use the motility to reflect the invasiveness of cancer cells, since these two

11 parameters are closely related and motility can be easily tracked in this setting. Consistent

12 with what has been reported previously, treatment with EGF significantly increased the

13 motility of MDA-MB-231 cells in fibrin gel (Fig. 3B). This is quantitively demonstrated

14 by differences in the 'mean migration speed', which refers to the mean migration distance

15 between 2 consecutive frames (Fig. 3C) (mean speeds of $1.74 \pm 0.91 \mu \mathrm{m} / 10 \mathrm{~min}$ for

$16+$ EGF vs $1.17 \pm 0.47 \mu \mathrm{m} / 10 \mathrm{~min}$ for control, $\mathrm{p}<0.01 * *)$. The mean MIs calculated from

175 min DHM measurements for these two cellular populations were also significantly

18 different (Fig. 3A, $0.248 \pm 0.140$ for the + EGF group vs $0.170 \pm 0.056$ for the control

19 group, $\mathrm{p}<0.01 * *)$.

20 From our results, it was also evident that there is significant heterogeneity in motility

21 within the fibrin gel at single cell level, which is independent of treatment with EGF.

22 This is not surprising as it has long been recognized that individual tumour cells respond

23 differently to environmental stimuli due to inherent cellular heterogeneity at the genetic,

24 epigenetic and phenotypic levels ${ }^{31,32}$. This heterogeneity also reflects the observation that

25 only a minority of highly invasive cells play central roles in tumour invasion ${ }^{31}$.

26 Therefore, having demonstrated the correlation between the 3D cellular motility and MI

27 at the population level, we then investigated whether single cell insights could be

28 obtained. Fig. 3D display the OT mapping, micromotion mapping and migration path of a

29 typical high MI cell in the EGF+ group while 3D-ii display those of a typical cell with a

30 low MI (the micromotion and migration movies of corresponding cells are shown in 
1 Movie S3-S6). We plotted the MIs of single cells in both the EGF treated (Fig. 3E-i) and

2 untreated (Fig. 3E-ii) groups vs their mean migration speeds determined over $12 \mathrm{~h}$ as

3 described above. Pearson correlation (r) analysis demonstrated a strong correlation

4 between the single cell micromotion and motility both for the EGF treated $(\mathrm{r}=0.764)$ and

5 EGF untreated (r=0.709) groups.

6 Subsequently, we investigated the mechanisms underlying the correlation between cell

7 motility and micromotion. More specifically, we studied the role of the actin cytoskeleton

8 in regulating cellular micromotion, which is known as a pivotal regulator of tumour cell

9 invasion. MDA-MB-231 cells were treated with a F-actin polymerization inhibitor

10 cytochalasin D, after which micromotion indexes were measured. OT images and

11 micromotion mappings of representative cells treated or not with cytochalasin D

12 (+cytochalasin D and control group) are shown in Fig. 3F. A clear reduction of the MI

13 was observed from the MI heat map of + cytochalasin D treated cell. Further statistical

14 comparison of MI between both groups (18 from control and 21 from treated group)

15 confirmed that the MI of cells was significantly reduced after cytochalasin D treatment

$16(0.172 \pm 0.076$ vs $0.122 \pm 0.033, \mathrm{P}<0.05$, Fig. $3 \mathrm{G})$. Measurements in an invasive prostate

17 cancer cell lines (PC-3) replicated the findings from the MDA-MB-231 model, although

18 the reduction of MIs in prostate cancer cells was less pronounced $(0.155 \pm 0.059$ vs 0.131

$19 \pm 0.033, \mathrm{P}<0.05$, Fig. S3). These sets of data support our hypothesis that the actin

20 cytoskeleton plays a significant role in regulating tumour cell micromotion as defined in

21 this work.

22 Acquisition of EMT phenotype results in increased cellular micromotion. The

23 acquisition of epithelial mesenchymal transition (EMT) phenotypes by tumour cells is

24 associated with disruption of cell-cell adhesion and polarity, modulation in tumour cell-

25 matrix adhesion, and remodelling of the cytoskeleton as well as with increased motility

26 and invasiveness ${ }^{33}$. We therefore endeavoured next to determine whether the acquisition

27 of an EMT phenotype is associated with increased optical cellular micromotion at the

28 single cell level as previously observed in $2 \mathrm{D}^{17,18}$. To address this question, we first

29 treated the MCF-7 cell line with an EMT induction medium for 5 days. As shown in Fig. 
1 S2, compared to the non-treated group the EMT induced MCF-7 cells displayed

2 significantly higher MIs $(0.219 \pm 0.097$ vs $0.128 \pm 0.043, \mathrm{P}<0.001 * * *)$, which confirmed

3 that the transition of MCF-7 cells to a more invasive phenotype also led to increased

4 micromotion. Next, to fully characterize how closely epithelial to mesenchymal

5 phenotype transition and micromotion are associated, the A549 VIM RFP cell line was

6 employed. This is a variant of the A549 cell line where vimentin is constitutively tagged

7 with the red fluorescent protein (RFP), thereby enabling direct monitoring with confocal

8 microscopy of the expression levels of this EMT marker. In this setting, we can use the

9 fluorescence intensity of individual A549 cells to determine relatively the degree of 10 phenotype transition. Consistent with the MCF-7 data, EMT induced A549 VIM RFP

11 cells displayed significant higher MI values over the control group (Fig. 4A, $0.192 \pm$

120.064 for the EMT group vs $0.123 \pm 0.033$ for the control group, $\mathrm{p}<0.001 * * *)$. As

13 expected and shown in Fig. 4B and C, the fluorescence intensities of EMT induced A549

14 VIM RFP cells were significantly higher than that of the untreated control group.

15 Next, we investigated whether the correlation exists at the single cell level. We plotted

16 the micromotion (measured with DHM) over their RFP fluorescence intensity (measured

17 with confocal microscopy) for every single cell, and calculated the correlation coefficient

18 between these two quantities. Typical data for the OT mapping, micromotion mapping

19 and fluorescence images of single cells from the EMT induction and control groups are

20 presented in Fig. 4D-ia to ic and 4D-iia to iic, respectively. The mean fluorescence

21 intensity of each cell was plotted vs the MI in Fig. 4E. From the data, a good correlation

22 was obtained with a Pearson correlation coefficients (r) between these 2 factors of 0.744.

23 Highly metastatic cells that display higher micromotion can be reduced by

24 migrastatic compounds. Next, we investigated whether tumour cells specifically

25 modified to display higher metastatic potential also exhibited higher micromotion within

26 a 3D environment. We have previously shown that miR-194 promotes prostate cancer

27 invasion and metastasis ${ }^{19}$. After being injected into the tail veins of mice, PC-3 cells

28 overexpressing miR-194 have a much greater capacity to colonise organs and growth as

29 assessed by whole-animal bio-luminescent imaging (Fig. 5A and B). To confirm this 
1 finding in an advanced in vitro setting, a microfluidic perfusable model of the human

2 microvasculature was used. In this model, live cancer cells can be perfused within the

3 'on-chip microvasculature' to investigate the trans-endothelial migration/extravasation.

4 This model has been previously successfully used to investigate the invasiveness of

5 different cell lines ${ }^{34-36}$. Fig. 5C shows typical images for the miR-194 overexpressing and

6 control groups $24 \mathrm{~h}$ after being perfused in the microvasculature. The extravasation ratios

7 (number of extravasated cells/total number of cells) were calculated from randomly

8 selected images. In agreement with the in vivo data, miR-194 overexpressing PC3 cells

9 displayed significant higher extravasation in comparison to the control group at $24 \mathrm{~h}$. A

10 trend for higher extravasation was also measured at 9h for the miR-194 over-expressing

11 group as shown in Fig. 5D. Subsequently, we measured the micromotion of miR-194

12 overexpressing PC-3 cells dispersed within the fibrin gel. MiR-194 over-expressing PC-3

13 cells displayed significantly higher micromotion indexes compared to that of the control

14 cells (Fig. 5E and F) (0.199 \pm 0.061 vs $0.153 \pm 0.041, \mathrm{p}<0.001 * * *)$, providing further

15 support that this measurement can be used as a proxy for invasion and metastasis.

16 Based on this data, we further investigated whether such methodology can be used to

17 evaluate the effects of anti-metastatic drugs that target cellular motility, namely

18 "migrastatic" compounds ${ }^{2,37}$, on cancer cells. To this end, we tested the effects of two

19 compounds known to inhibit the motility of prostate cancer cells ${ }^{20,21}$, genistein and KBU

20 2046, on micromotion. Specifically, PC-3 cancer cells were encapsulated in fibrin gel and

21 equilibrated in an incubator for $6 \mathrm{~h}$, then the culture medium in the 3D culture chamber

22 was replaced with fresh medium (control), the medium containing either genistein or

23 KBU 2046. Micromotion indexes were measured before, 24h, and 48h after treatment.

24 The data for PC-3 cellular MIs treated by genistein or KBU 2046 are presented in Fig. 5G

25 and $\mathrm{H}$ and control in Fig S4, respectively. Significant reductions of PC-3 cellular MIs

26 were observed in the genistein and KBU 2046 treatment groups but was not observed in

27 control, indicating that the reduction of MIs were induced by migrastatics rather than by

28 other environmental factors. Interestingly, the micromotion variation dynamics of these

29 two compounds are different. Significant reduction of MI was observed after $24 \mathrm{~h}$ of

30 genistein treatment $(0.158 \pm 0.053$ vs $0.139 \pm 0.058, \mathrm{P}<0.05)$, but $\mathrm{MI}$ recovered back to 
1 the before treatment level $(0.158 \pm 0.053$ vs $0.154 \pm 0.077)$ after $48 \mathrm{~h}$. In contrast, KBU

22046 continuously reduced the MIs of PC-3 over the entire duration of the experiment.

3 Although the inhibition effect of KBU 2046 to MIs at 24h after treatment is not

4 significant $(0.165 \pm 0.071$ vs $0.145 \pm 0.045, \mathrm{P}=0.06)$, a reduction in $\mathrm{MI}$ was observed

$5 \quad(0.165 \pm 0.071$ vs $0.133 \pm 0.046, \mathrm{P}<0.01)$ after $48 \mathrm{~h}$ KBU 2046 treatment with higher

6 significance compared to genistein induced MI reduction. Figure 5I plots the fold

7 changes of mean MIs of PC-3 at different time points with or without KBU-2046 or

8 genistein treatment. The value in each square equals to the ratio of mean MIs between at

9 certain time points and at before treatment ( e.g. $\mathrm{KBU}_{24 \mathrm{~h}}=\overline{\mathrm{MI}}_{\mathrm{KBU} @ 24 \mathrm{~h}} / \overline{\mathrm{MI}}_{\mathrm{KBU} @ \mathrm{Before}}$ ). This

10 plot illustrates the trend that KBU2046 and genistein exert similar effects at $24 \mathrm{~h}$ but

11 distinctive at $48 \mathrm{~h}$, in which time point the MIs of genistein treated group recovered to

12 before treatment level while that of KBU treatment group reduced further. This set of

13 data, particularly the recovery of MIs at $48 \mathrm{~h}$ after genistein treatment, might also suggest

14 PC-3 are of higher tolerance to genistein compared to KBU 2046. This is consistent with

15 the observation from Li et. al that KBU 2046 is more effective in inhibition metastasis of 16 prostate cancer cells than genistein ${ }^{20}$.

\section{Discussion}

18 Tumour cell invasion of nearby tissues is one of the most important processes during 19 cancer progression. Therefore, the identification of specific factors that can 20 activate/suppress tumour cell invasiveness has strong potential to provide not only an 21 improved mechanistic understanding of the metastatic process but also to assist in the 22 development of novel therapeutic approaches. Importantly, solid tumours are inherently 23 heterogeneous, being comprised of cellular subpopulations with vast differences in gene 24 and protein expression, tumour-forming ability, and invasiveness ${ }^{38-40}$. In addition, a 25 defining characteristic of tumour cells invasiveness is the fact that it inherently occurs in 26 a 3D environment and over a relatively long period of time. Current approaches to 27 determine the motility of tumour cells typically involve measuring their actual movement 28 in space, which suffer from several limitations, especially when performed in a 3D 29 environment ${ }^{7-9}$. 
1 We report here a new paradigm, which is that measurement at the single cell level of an

2 optical cellular micromotion index calculated from the dynamic nanoscale fluctuations of

3 the OT is strongly correlated to intracellular biological processes underpinning cellular

4 motility through ECM-like gel. The practical implementation of this concept enables the

5 measurement, within a few minutes and non-invasively, of the invasiveness of single

6 tumour cells within a 3D environment that mimics that of real tumour. The concept of

7 cellular micromotion was initially demonstrated using impedance-based measurement of

8 the nanoscale fluctuations of the distance between cell membranes and a gold electrode.

9 Impedance based micromotion could be used to identify distinctive micromotion features

10 of cancerous cells from normal cells. In addition, tumour cell lines with higher

11 invasiveness were also shown to have higher micromotion (e.g. MDA-MB-231 vs MCF-

12 7) ${ }^{15}$ This is in agreement with our observation that the optical cellular micromotion

13 indexes of MDA-MB-231 cells were significantly higher than that calculated for the less

14 invasive MCF-7 cells $\left(0.170 \pm 0.06\right.$ vs $0.128 \pm 0.04, \mathrm{p}<0.01^{* *}$ at $6 \mathrm{~h}$ after seeding and

$150.165 \pm 0.05$ vs $0.113 \pm 0.04, \mathrm{p}<0.001 * * *$ at $24 \mathrm{~h}$ after seeding, Table. S1). However,

16 impedance-based micromotion measurement is limited by the inherent requirement for

17 the cells to be adhered on an aberrantly rigid electrode. In addition, while single cell

18 measurements are theoretically possible, practical implementation requires the formation

19 of a cellular monolayer onto the electrode and therefore the correlation of micromotion

20 and invasiveness at the single cancer cell level has never been shown. While the

21 impedance-based micromotion is an indirect measurement of the dynamic fluctuations of

22 the cell membranes, the optical cellular micromotion as defined in this report is based on

23 directly measuring the dynamic dry mass reorganisation that occurs within live cells. This

24 reorganisation is measured as spatiotemporal fluctuations in the local OT (proportional to

25 the cellular dry mass) $)^{41}$ which can be mapped with digital holographic microscopy in a

26 high precision manner. Several previous studies reported that the cellular dry mass

27 redistribution is driven by certain receptor activation ${ }^{42,43}$. In particular, EGF stimulation

28 was reported to mediate dynamic mass redistribution in epidermal growth factor receptor

29 (EGFR) positive cancer cells ${ }^{44,}$. This is consistent with our observation that EGF 
1 upregulated the MI and migration capability of MDA-MD-231, although the cellular

2 environment, as well as the objectives and analysis tools are very different between these

3 two studies.

4 The "micromotion" as defined and measured in this study likely represents a composite 5 of multiple intracellular processes that manifest as a single optically detectable cellular 6 phenomenon, and presumably some cellular processes included in the observation and 7 quantification are either less relevant or even irrelevant to cell migration/invasion. This 8 "black box" aspect is the limitation of most label free observations ${ }^{42}$. To better interpret 9 the measured "optical cellular micromotion", the key regulators of micromotion and the 10 mechanism behind such correlation between optical cellular micromotion and invasion 11 were explored in this study. The key hypothesis here is that one or several intracellular 12 machineries that drives cellular invasion overlaps with the optical micromotion 13 measurements. It is well established that tumour cells actively migrate within tissues 14 through dysregulation of cytoskeletal components such as actin dynamics and 15 organization $^{46-48}$. Our mechanistic data confirmed that the actin cytoskeleton is a 16 significant regulator of optical cellular micromotion, supporting our speculation with 17 respect to the mechanism behind the correlation.

18 Interestingly, we found that $\sim 5 \mathrm{~min}$ of micromotion measurement can effectively 19 distinguish cancer cells with different motility/invasiveness. This time span is much 20 shorter than that of standard invasion assays based on measuring accumulative effects of 21 invasion, such as distance or porous membrane translocation. This is not surprising, 22 especially considering the dynamics of cytoskeleton activity in 3D environment. In fact, 23 cytoskeletal dynamics that directly or indirectly link to cell invasion occur within similar 24 time scale, including the displacement of actin filament (136s in a migrating keratocyte ${ }^{49}$ ) 25 or microtubule redistribution (120s in human fibrosarcoma cells embedded in collagen I 26 matrices $^{50}$ ). A typical example is the dynamics of cellular protrusion that plays a crucial 27 role in mediating ECM degradation and tumour cell invasion, such as lamellipodia, 28 invadopodia or bleb. The formation and translocation of these actin and/or other 29 cytoskeleton components abundant subcellular structures involve a series of transient, 
1 localized remodelling of the cell membrane and intracellular structures. For example, the

2 formation of invadopodia involves vast cell membrane ruffling, extension and protrusion

3 with only a few minutes lifetime ${ }^{51}$. These processes eventually result in rapid cell

4 morphological changes ${ }^{52-54}$ and associated cellular dry mass redistribution.

5 It also worth noting that cells in the 3D environment were found to have significantly

6 higher micromotion index than that in 2D (Fig. 2C). This is not surprising when

7 considering the inherent differences between 2D and 3D environments, which change the

8 modes of cell-matrix interaction and in turn influence cellular behaviours. For example,

9 focal adhesions of cells growing in a 2D setting are substantially larger in scale $(\sim 15 \mu \mathrm{m}$

10 vs $\sim 0.3 \mu \mathrm{m})$ and more stably formed ( $15 \mathrm{~min}$ vs $\sim 1 \mathrm{~s}$ of lifetime) than in $3 \mathrm{D}^{6}$. This

11 distinction not only dictates the different motility of cancer cells ${ }^{3}$, but also could

12 contribute to the substantial difference in micromotion for cells in $2 \mathrm{D}$ vs $3 \mathrm{D}$ observed in

13 this study. Indeed, the substantially shorter lifetime of focal adhesion suggests that

14 subcellular structures are reorganized far more rapidly in a 3D environment, which is

15 expected to yield higher amount of micromotion within a period of time than cells in 2D.

16 One important aspect of the micromotion concept is its ability to inform about the

17 heterogeneity within the tested population with respect to invasiveness as well as invasive

18 phenotype (Fig. 3E and 4D). Tumours display high level of heterogeneity and potentially

19 develop resistance to therapeutics with different mechanisms and in different degrees ${ }^{31}$.

20 This heterogeneity can present in individual level, organ level (primary tumour vs

21 metastasis), or intra-tumour level ${ }^{55}$. Therefore, indication of the killing (e.g. cytotoxic) or

22 inhibition (e.g. cytostatic or migrastatic) effects towards such small portion of cells

23 within the population is important in anti-tumour therapeutics screening and validation ${ }^{40}$,

$24 \quad 56$. Current population-based invasion measurements are often blind to important cellular

25 subpopulations with high invasiveness, which likely negatively impact on their relevance

26 to the in vivo situation, and the capabilities to evaluate the effectiveness of migrastatic

27 compounds.

28 Being able to evaluate migrastatic drug efficacy is a key advantage of optical cellular 29 micromotion. In contrast to conventional cytotoxic or cytostatic drugs that targeting the 
1 viability or growth of cancer cells, migrastatic drugs target the migration, invasion, and

2 metastasis of cancer cells ${ }^{2}$. By specifically targeting cytoskeletal components of cancer

3 cells, the small molecules disrupt machineries that play key roles in cancer cells invasion

4 (e.g. cytoskeletal dynamics, lamellipodia/invadopodia formation/deformation, cell

5 contraction/cell rear retraction) $)^{57}$. One example is KBU 2046, which decreases the

6 motility of prostate cancer cells by modulating the activity of the oncoprotein HSP90.

7 Our micromotion measurements support the hypothesis that KBU 2046 inhibits prostate

8 cancer cell motility through disrupting the cytoskeleton reorganization, considering the

9 association between micromotion and cytoskeleton activities. Also, optical cellular

10 micromotion measurements provide insights into migrastatic compounds time response

11 on both motility and cytoskeleton activity of single cells. Taken together our results show

12 the feasibility of using optical cellular micromotion measurements to assess the efficacy

13 of candidate migrastatic drugs. It also suggests that it could be used to monitor transient

14 invasiveness changes of cancer cells at specific time point during drug treatment, which

15 is not possible in standard invasion assay but could be very useful towards yielding better

16 understanding of the mechanisms underpinning cancer motility inhibition.

17 A number of limitations in this study should be considered and warrant future 18 investigation. First, although cells were suspended in fibrin gel that support cellular 19 motility in 3D, the ECM biological and structural complexity (e.g. complex ECM 20 bundles, spatial organization of ECM fibers) is not fully recapitulated by fibrin. The 21 specific structure of the ECM regulates tumour cells motilities through driving the 22 dynamic re-organization of cytoskeleton and focal adhesion. ${ }^{58,59}$ Micromotion indexes 23 measured in more complex gels that better mimic the native ECM of specific tissues is 24 therefore anticipated to be of higher physiological relevance and consequently could 25 yield better biological insight. In addition, only isolated single cells with clear cell 26 boundaries were included in the micromotion measurements in the present study as this 27 significantly reduces the technical difficulties in image acquisition and processing (e.g. 28 image segmentation). However, cell-cell interactions play a significant role in the 29 migration of cells through tissues ${ }^{60,61}$ and refinement of the method to enable such 30 complex measurement could further strengthen its significance. 
bioRxiv preprint doi: https://doi.org/10.1101/2021.08.26.457857; this version posted August 27, 2021. The copyright holder for this preprint (which was not certified by peer review) is the author/funder. All rights reserved. No reuse allowed without permission. 


\section{Materials and methods}

2 Materials. Phosphate buffer saline (PBS), 4\% formalin solution, fibrinogen and thrombin from

3 bovine plasma were all purchased from Sigma-Aldridge. Poly(dimethylsiloxane) (PDMS) was

4 acquired from Dow Corning (Singapore). Cytochalasin D was also obtained from Sigma-Aldridge

5 and dissolved in DMSO as stock solution. DMEM ( $\mathrm{pH}=7.4$, Sigma-Aldridge) for MDA-MB-

6231 and MCF-7 breast cancer cell lines culture and F-12K (ATCC) for A549 VIM-RFP culture

7 were supplemented with $10 \%$ fetal bovine serum (FBS, Gibco) solution and $1 \%$ antibiotics

8 (Gibco). Both normal PC-3 prostate cancer cell line (ATCC) and PC-3 transfected with miR-194

9 or negative control mimic were maintained in RPMI-1640 medium (Gibco) supplemented with 10

$10 \%$ fetal bovine serum (FBS, Gibco) solution and $1 \%$ antibiotics (Gibco). Human umbilical vein

11 endothelial cells (HUVECs) and normal human lung fibroblasts (NHLFs) were purchased from

12 Lonza and cultured in EGM-2 and FGM-2 (Lonza) supplemented with EGM-MV and FGM-2

13 Bullet Kit, respectively. Human epidermal growth factor (hEGF) was purchased from Lonza and

14 EMT inducing media supplement was purchased from R\&D System.

15 Cell Culture. MDA-MB-231, MCF-7 breast cancer cell lines, A549 VIM-RFP cancer cell line

16 and PC-3 prostate cancer cell line with or without transfection (ATCC) used in this study were

17 cultured in $75 \mathrm{~mm}^{2}$ tissue culture flasks and maintained in $37{ }^{\circ} \mathrm{C}$ and $5 \% \mathrm{CO}_{2}$ humidifying

18 incubator. Medium was changed every 2-3 days and cells were subcultured until reach 70-80\%

19 confluency. For DHM imaging, a custom-made device (imaging device) was used to culture cell

20 both in 2D and 3D. A PDMS chamber with 2 openings for injecting medium was made with both

21 sides sealed by optically clear polymer coverslips (Ibidi), in order to make both sides flat and

22 optically transparent for DHM imaging. The distance between the two coverslips was $300 \mu \mathrm{m}$.

23 For 2D cell culture, the cells were seeded directly into the chamber after both sides have been

24 sealed. A tissue culture treated polymer coverslip at the bottom was used. For 3D cell culture,

25 fibrin gel was used for extracellular matrix. Only one side of chamber was sealed with a coverslip

26 and the other side was left unsealed before cell seeding. Subsequently $5 \mu \mathrm{L}$ of cell suspension

27 with $5 \mathrm{U} / \mathrm{mL}$ thrombin and $5 \mu \mathrm{L}$ of $10 \mathrm{mg} / \mathrm{mL}$ fibrinogen solution was homogeneously mixed. 5

$28 \mu \mathrm{L}$ of the cell/fibrin gel mixture was dropped onto the coverslip in the PDMS chamber which was

29 sealed with another coverslip. After 10 minutes when the fibrin gel completely gelled, cell culture

30 medium was injected into the PDMS chamber. In both experiments the final concentrations of

31 cells (in medium, or in pre-gel solution) were $5 \times 10^{6} / \mathrm{mL}$. 
1 Micromotion index measurement. In this study, a commercially available DHM® T-1000

2 (Lyncée Tech) was used. The custom-made device for cell culture was kept in a stage-top 3 incubator (Chamlide) maintained at $37{ }^{\circ} \mathrm{C}$ and $5 \% \mathrm{CO}_{2}$ during the real time DHM imaging. Koala

4 software native to the DHM was used to control the imaging and reconstructed holographic 5 images to phase images. The software allows automated conversion of raw phase images into OT

6 measurements. It is worth noting here that practically what is being measured with the DHM is 7 the phase shift of the light that passes through a cell but it is proportional to the cell optical 8 thickness (OT). In this sense we refer to "phase shift" when we discuss light or optics but "OT" 9 when we refer to cells.

10 For micromotion measurement, 1024 images were typically acquired every $250 \mathrm{~ms}$ with the $20 \times$ 11 magnification objective. In the case of 3D culture, each cells were digitally focussed by adjusting 12 the "focus" parameter from "reconstruction settings" window in the Koala software. In this case 13 new region of interest (ROI) was defined and the in-focus cell was included in the reconstructed 14 phase images. Reconstructed phase images with single focused cell rather than raw phase images 15 that include both in-focus and out-of-focus cells, were used for micromotion measurement in the 16 next step and were presented in the figures. Since we were interested in the optical cellular 17 micromotion of the single cells, in the study only individual cells with clear cell boundary were 18 included in the analysis. Clusters of cells or cells overlapped with others were removed from 19 analysis. In addition, in 3D cell measurement, cells with average OT lower than average value of $202 \mathrm{D}$ cells and has typical 2D morphology were considered as 2D cells and were removed from 21 analysis.

22 A mask for each cell is first built to segment cells from the background automatically by an 23 algorithm using a threshold determined from pixel height. We used the method reported by 24 Shaked et. al to quantify the dynamic OT (OT) fluctuation profile. In brief, we define the shift of 25 OT $(\triangle \mathrm{OT})$ using eq $(1)$ :

$$
\Delta O T_{(x, y)}=O T_{(t, x, y)}-\left\langle O T_{(t, x, y)}\right\rangle_{t}
$$

27 in which $\mathrm{OT}_{(\mathrm{t}, \mathrm{x}, \mathrm{y})}$ refers to spatially variant $\mathrm{OT}$ of pixel $(\mathrm{x}, \mathrm{y})$ at time point $\mathrm{t}$ and $\left\langle\mathrm{OT}_{(\mathrm{t}, \mathrm{x}, \mathrm{y})}\right\rangle_{\mathrm{t}}$ refers 28 to the average of $\mathrm{OT}_{(\mathrm{t}, \mathrm{x}, \mathrm{y})}$ over the entire imaging period. Then we calculate the mean square of $29 \Delta$ OT using eq (2): 
5

$$
\Delta O T_{v a r}(x, y)=\frac{1}{N} \sum_{t l=1}^{N}(\Delta O T(l i, x, y))^{2}
$$

in which $i=[1, \ldots, 1024]$ is indexing the number of images. Lastly we define the MI with eq (3):

$$
M I=\overline{M I(x, y)}=\sqrt{\left\{\Delta O T_{\text {vpur }}(x, y)\right\}_{x, y}}
$$

in which the MI of every pixel in a cell mask is calculated then averaged over the whole mask

Cell random migration assay and speed calculation. MDA-MB-231 breast cancer cells were used for random migration assay. After 5 min imaging with DHM to obtain the MIs, a $12 \mathrm{~h}$ DHM imaging was carried out, in which 72 images were acquired every 10 min using the $10 \times$ magnification objective. The Trackmate plugin in open access software Fiji was then used to automatically segment and track the cell movement and record the position of cell centre (x,y) in every frame. The mean displacement between each frame was calculated with eq (4)

$$
\bar{d}=\frac{1}{N} \sum_{i=1}^{N} \sqrt{\left(x_{t+1}-x_{i}\right)^{2}+\left(y_{l+1}-y_{l}\right)^{2}}
$$

in which $i=[1, \ldots, 72]$ is indexing the number of images. Mean speed (v) equals to the /frame.

Actin cytoskeleton inhibition. Both MDA-MB-231 and PC-3 were used for action cytoskeleton inhibition assay. Actin cytoskeleton inhibition was performed directly in 3D environment. In specific, $1 \mathrm{mg} / \mathrm{mL}$ cytochalasin D stock solution that dissolved in DMSO was 1:200 diluted in 6 $\mathrm{mg} / \mathrm{mL}$ fibrinogen solution, which then quickly mixed with same volume of cell suspension and seeded in the device. The cell seeding procedures followed the 3D cell culture protocol described in previous cell culture section. In this case the 3D fibrin gel was supplemented with $2.5 \mu \mathrm{g} / \mathrm{mL}$ cytochalasin D. In the control group DMSO at final concentration of $0.4 \%(\mathrm{v} / \mathrm{v})$ was supplemented. Then device was filled up with cell culture medium containing $2.5 \mu \mathrm{g} / \mathrm{mL}$ 
1 cytochalasin D or $0.4 \%$ DMSO and kept in incubator for $2 \mathrm{~h}$ for equilibration before micromotion 2 measurement.

3 EMT induction. MCF-7 and A-549 was used for EMT induction assay. EMT induction was 4 performed on 2D according to the protocol provided by manufacturer, then encapsulated in gel 5 for $3 \mathrm{D}$ measurements. Briefly, $1 \times 10^{5}$ cells were seeded in 6 well plate with $2 \mathrm{~mL}$ of the culture 6 medium supplemented with 1\% StemXVivo EMT inducing media supplement. The medium was 7 replaced with fresh EMT induction medium on day 3 after induction. After 5 days the EMT 8 induction is completed and cells are used as described above for DHM imaging. Imaging of VIM9 RFP reporter was performed using LSM 710 confocal microscope (Zeiss, Germany) and 10 quantification of fluorescent intensity of single A549 VIM-RFP cell was performed using ImageJ 11 (NIH). Single fluorescent cells were segmented from background manually for determining of 12 area $\left(\mu \mathrm{m}^{2}\right)$ and fluorescence intensity (F.I.) was measured automatically by ImageJ. Mean 13 fluorescence intensity (M.F.I.) was calculated using F.I divided by area of single cells (M.F.I.= 14 F.I. / area).

15 MiRNA transfection and intravenous metastasis assay. Both the transfection and intravenous 16 metastasis assay were conducted following the protocols reported previously ${ }^{19}$ without any 17 modification. Intravenous metastasis assay were approved by the University of Adelaide Animal 18 Ethics Committee (approval number M-2014-180C).

19 Microvasculature-on-a-chip preparation. The preparation of microvasculature-on-a-chip was 20 performed following the protocol developed by Chen et. $\mathrm{al}^{35}$ with slight modification as we 21 previously reported ${ }^{62}$. Briefly, HUVECs $\left(2.5 \times 10^{7} / \mathrm{mL}\right)$ and NHLFs $\left(1.2 \times 10^{7} / \mathrm{mL}\right)$ were suspended 22 in endothelial culture medium supplemented with $4 \mathrm{U} / \mathrm{mL}$ thrombin and mixed with equal 23 volume of $6 \mathrm{mg} / \mathrm{mL}$ fibrinogen solution. After quickly pipetting, cells-gel mixture was injected 24 into the corresponding cell channel and incubated by approximately $10 \mathrm{~min}$ for the gelation of 25 fibrinogen. Medium in the device was changed every day until the formation of well-formed 26 microvasculature (approximately 5 days).

27 Extravasation assay. The tumour cell extravasation assay was performed after 5 days when the 28 microvasculature is well-formed and more than 50\% of interpost regions (regions in between the 29 device micropillars) have established vascular openings. For extravasation assay, the medium was 30 withdrawn from all the reservoirs and a $50 \mu \mathrm{L}$ of control or miR-overexpressed PC-3 cells 31 suspension with a concentration $2 \times 10^{5} / \mathrm{mL}$ was pipetted into one reservoir linked to the medium 
1 channel that flanks the HUVEC (microvasculature) channel. The medium was allowed to perfuse

2 across the medium channel gradually to fill up the reservoir on the other side of medium channel,

3 driven by hydrostatic pressure. After reaching equilibrium of hydrostatic pressure, another $50 \mu \mathrm{L}$

4 of cell suspension was added into both reservoirs on one side to establish a hydrostatic pressure

5 across the microvasculature channel and the device was placed in the incubator. After $10 \mathrm{~min}$,

6 when cancer cells were fully perfused across the microvasculature, the cell suspension was

7 withdrawn, and fresh medium was added to all the reservoirs to wash away cancer cells not

8 attached to the medium channel. Finally, the number of perfused and arrested cells were observed

9 under bright field microscopy to ensure there are enough cells perfused and arrested into

10 microvasculature (typically around 100-200 cancer cells per device). The extravasation rate was

11 calculated as the proportion of cancer cells that escaped from the microvasculature relative to the

12 total number of cells that remained inside the microvasculature on chip device. Identification of

13 cancer cells that escaped from microvasculature was performed using confocal microscopic

14 images (LSM 710 confocal microscope). Boundary of microvasculature was visualized by

15 endothelial marker (VE-cadherin) labelled with standard immunostaining method, PC-3 with or

16 without overexpression of miR-194 were visualized by self-tagged fluorescence.

17 "Migrastatic" compound treatment. KBU 2046 is kindly provided by Raymond Bergan's

18 laboratory and preparation of KBU 2046 cell treatment solution follows the protocol provided by

19 Raymond Bergan's laboratory. Briefly, $5 \mathrm{mg}$ of KBU 2046 was dissolved in $206.4 \mu \mathrm{L}$ to prepare

$20100 \mathrm{mM}$ stock solution, then aliquoted $10 \mu \mathrm{L}$ such solution in $1.5 \mathrm{~mL}$ Eppendorf tube and

21 preserved in $-20 \square$. Before use, stock solution was kept in $37 \square$ incubator until the solution was

22 completely thawed and $90 \mu \mathrm{L}$ additional DMSO was added into tube to prepare $10 \mathrm{mM}$ solution.

23 Then the solution was 1:1000 diluted in cell culture medium to obtain $10 \mu \mathrm{M}$ working solution.

24 Both $10 \mathrm{mM}$ DMSO solution and $10 \mu \mathrm{M}$ working solution will be disposed immediately after

25 experiment and will not be reused. Genistein was purchased from Sigma and similar protocol was

26 followed to prepare both stock and working solution with only modification that dissolving $5 \mathrm{mg}$

27 genistein in $185.0 \mu \mathrm{L}$ DMSO to prepare $100 \mathrm{mM}$ stock solution. For in-device cell treatment

28 experiment, cells were seeded in fibrin gel in device and incubated for $6 \mathrm{~h}$ before DHM imaging.

29 After imaging completed, the device was placed back to incubator for at least 15 min before

30 performing medium replacement. For the medium replacement, a drop of fresh medium (with or

31 without drugs) was placed on top of both inlet and outlet with $1 \mathrm{~mL}$ pipette to wet the PDMS

32 surrounding the inlet and outlet, followed by removing most of medium on outlet and adding 
1 medium on inlet. Driven by hydrostatic force, the flow of medium from inlet to outlet will form,

2 and then keep removing medium on outlet to continue the flow until most of medium has flowed

3 through the device. Repeat such procedures at least twice to make sure most medium in the

4 chamber has been replaced with fresh medium.

5 Statistical analysis. Statistical analyses were performed using student t-test for any data set 6 comparison. $\mathrm{P}<0.05$ was considered to be statistically significant.

\section{Acknowledge}

8 This work was performed in part at the South Australian node of the Australian National

9 Fabrication Facility, a company established under the National Collaborative Research

10 Infrastructure Strategy to provide nano and microfabrication facilities for Australia's

11 researchers. We thank Prof. Raymond Bergan and his laboratory from Oregon Health \&

12 Science University to provide KBU 2046 compound and relevant documents. This work

13 was supported by the ARC Centre of Excellence in Convergent Bio and Nano Science

14 and Technology and funding from the National Health and Medical Research Council of

15 Australia (ID 1083961 to LAS). LAS is supported by a Principal Cancer Research

16 Fellowship awarded by Cancer Council's Beat Cancer project on behalf of its donors, the

17 state Government through the Department of Health and the Australian Government

18 through the Medical Research Future Fund.

\section{References}

20 1. Scheidt, K. et al. Synthesis and Biological Evaluation of 3-Arylindazoles as Selective MEK4 Inhibitors. ChemMedChem (2019).

2. Gandalovičová, A. et al. Migrastatics - anti-metastatic and anti-invasion drugs: promises and challenges. Trends in cancer 3, 391-406 (2017).

3. Wirtz, D., Konstantopoulos, K. \& Searson, P.C. The physics of cancer: the role of physical interactions and mechanical forces in metastasis. Nature Reviews Cancer 11, 512-522 (2011).

4. Friedl, P. \& Alexander, S. Cancer invasion and the microenvironment: plasticity and reciprocity. Cell 147, 992-1009 (2011).

5. Friedl, P., Sahai, E., Weiss, S. \& Yamada, K.M. New dimensions in cell migration. Nature reviews Molecular cell biology 13, 743 (2012). 
6. Fraley, S.I. et al. A distinctive role for focal adhesion proteins in threedimensional cell motility. Nature cell biology 12, 598-604 (2010).

7. Conway, J.R., Carragher, N.O. \& Timpson, P. Developments in preclinical cancer imaging: innovating the discovery of therapeutics. Nature Reviews Cancer 14, 314 (2014).

8. Echeverria, V. et al. An automated high-content assay for tumor cell migration through 3-dimensional matrices. Journal of biomolecular screening 15, 11441151 (2010).

9. Burgstaller, G., Oehrle, B., Koch, I., Lindner, M. \& Eickelberg, O. Multiplex profiling of cellular invasion in 3D cell culture models. PloS one 8, e63121 (2013).

10. Hood, J.D. \& Cheresh, D.A. Role of integrins in cell invasion and migration. Nature Reviews Cancer 2, 91 (2002).

11. Yamaguchi, H. \& Condeelis, J. Regulation of the actin cytoskeleton in cancer cell migration and invasion. Biochimica et Biophysica Acta (BBA)-Molecular Cell Research 1773, 642-652 (2007).

12. Giaever, I. \& Keese, C.R. Micromotion of mammalian cells measured electrically. Proceedings of the National Academy of Sciences 88, 7896-7900 (1991).

13. Tarantola, M. et al. Dynamics of human cancer cell lines monitored by electrical and acoustic fluctuation analysis. Integrative Biology 2, 139-150 (2010).

14. Yang, C.-T., Méjard, R.g., Griesser, H.J., Bagnaninchi, P.O. \& Thierry, B. Cellular micromotion monitored by long-range surface plasmon resonance with optical fluctuation analysis. Analytical chemistry 87, 1456-1461 (2015).

15. Parekh, A. et al. Bioimpedimetric analysis in conjunction with growth dynamics to differentiate aggressiveness of cancer cells. Scientific reports $\mathbf{8}, 783$ (2018).

16. Lovelady, D.C., Richmond, T., Maggi, A.N., Lo, C.-M. \& Rabson, D.A. Distinguishing cancerous from noncancerous cells through analysis of electrical noise. Physical Review E 76, 041908 (2007).

17. Schneider, D., Tarantola, M. \& Janshoff, A. Dynamics of TGF- $\beta$ induced epithelial-to-mesenchymal transition monitored by electric cell-substrate impedance sensing. Biochimica et Biophysica Acta (BBA)-Molecular Cell Research 1813, 2099-2107 (2011).

18. Baronsky, T. et al. Cell-substrate dynamics of the epithelial-to-mesenchymal transition. Nano letters 17, 3320-3326 (2017).

19. Das, R. et al. MicroRNA-194 promotes prostate cancer metastasis by inhibiting SOCS2. Cancer research 77, 1021-1034 (2017).

20. $\mathrm{Xu}, \mathrm{L}$. et al. Precision therapeutic targeting of human cancer cell motility. Nature communications 9, 2454 (2018).

21. Lakshman, M. et al. Dietary genistein inhibits metastasis of human prostate cancer in mice. Cancer research 68, 2024-2032 (2008).

22. Herrick, S., Blanc-Brude, O., Gray, A. \& Laurent, G. Fibrinogen. The international journal of biochemistry \& cell biology 31, 741-746 (1999).

23. Liu, J. et al. Soft fibrin gels promote selection and growth of tumorigenic cells. Nature materials 11, 734 (2012). 
24. Yamauchi, T., Iwai, H. \& Yamashita, Y. Label-free imaging of intracellular motility by low-coherent quantitative phase microscopy. Optics Express 19, 55365550 (2011).

25. Bagnaninchi, P.O., Drummond, N., Holmes, C., Daoud, J. \& Tabrizian, M. Twodimensional and three-dimensional viability measurements of adult stem cells with optical coherence phase microscopy. Journal of Biomedical Optics 16, 086003 (2011).

26. Shaked, N.T., Satterwhite, L.L., Bursac, N. \& Wax, A. Whole-cell-analysis of live cardiomyocytes using wide-field interferometric phase microscopy. Biomedical optics express 1, 706-719 (2010).

27. Griffith, L.G. \& Swartz, M.A. Capturing complex 3D tissue physiology in vitro. Nature reviews Molecular cell biology 7, 211 (2006).

28. Pampaloni, F., Reynaud, E.G. \& Stelzer, E.H. The third dimension bridges the gap between cell culture and live tissue. Nature reviews Molecular cell biology $\mathbf{8}$, 839 (2007).

29. Meyer, A.S. et al. 2D protrusion but not motility predicts growth factor-induced cancer cell migration in 3D collagen. J Cell Biol 197, 721-729 (2012).

30. Price, J.T., Tiganis, T., Agarwal, A., Djakiew, D. \& Thompson, E.W. Epidermal growth factor promotes MDA-MB-231 breast cancer cell migration through a phosphatidylinositol $3^{\prime}$-kinase and phospholipase C-dependent mechanism. Cancer research 59, 5475-5478 (1999).

31. Meacham, C.E. \& Morrison, S.J. Tumour heterogeneity and cancer cell plasticity. Nature 501, 328-337 (2013).

32. Easwaran, H., Tsai, H.-C. \& Baylin, S.B. Cancer epigenetics: tumor heterogeneity, plasticity of stem-like states, and drug resistance. Molecular cell 54, 716-727 (2014).

33. Thiery, J.P. Epithelial-mesenchymal transitions in tumour progression. Nature Reviews Cancer 2, 442 (2002).

34. Jeon, J.S. et al. Human 3D vascularized organotypic microfluidic assays to study breast cancer cell extravasation. Proceedings of the National Academy of Sciences 112, 214-219 (2015).

35. Chen, M.B. et al. On-chip human microvasculature assay for visualization and quantification of tumor cell extravasation dynamics. Nature protocols 12, 865 (2017).

36. Chen, M.B., Lamar, J.M., Li, R., Hynes, R.O. \& Kamm, R.D. Elucidation of the roles of tumor integrin $\beta 1$ in the extravasation stage of the metastasis cascade. Cancer research 76, 2513-2524 (2016).

37. Rosel, D., Fernandes, M., Sanz-Moreno, V. \& Brábek, J. Migrastatics: Redirecting R\&D in Solid Cancer Towards Metastasis? Trends in Cancer 5, 755756 (2019).

38. Bedard, P.L., Hansen, A.R., Ratain, M.J. \& Siu, L.L. Tumour heterogeneity in the clinic. Nature 501, 355 (2013).

39. Turner, N.C. \& Reis-Filho, J.S. Genetic heterogeneity and cancer drug resistance. The lancet oncology 13, e178-e185 (2012). 
40. Marusyk, A., Almendro, V. \& Polyak, K. Intra-tumour heterogeneity: a looking glass for cancer? Nature Reviews Cancer 12, 323-334 (2012).

41. Zangle, T.A. \& Teitell, M.A. Live-cell mass profiling: an emerging approach in quantitative biophysics. Nature methods 11, 1221 (2014).

42. Schröder, R. et al. Applying label-free dynamic mass redistribution technology to frame signaling of $\mathrm{G}$ protein-coupled receptors noninvasively in living cells. Nature protocols 6, 1748-1760 (2011).

43. Codd, E.E., Mabus, J.R., Murray, B.S., Zhang, S.-P. \& Flores, C.M. Dynamic mass redistribution as a means to measure and differentiate signaling via opioid and cannabinoid receptors. Assay and drug development technologies 9, 362-372 (2011).

44. Du, Y. et al. Distinct growth factor-induced dynamic mass redistribution (DMR) profiles for monitoring oncogenic signaling pathways in various cancer cells. Journal of Receptors and Signal Transduction 29, 182-194 (2009).

45. Fang, Y., Ferrie, A.M., Fontaine, N.H. \& Yuen, P.K. Characteristics of dynamic mass redistribution of epidermal growth factor receptor signaling in living cells measured with label-free optical biosensors. Analytical chemistry 77, 5720-5725 (2005).

46. Sanz-Moreno, V. \& Marshall, C.J. The plasticity of cytoskeletal dynamics underlying neoplastic cell migration. Current opinion in cell biology 22, 690-696 (2010).

47. Cummins, T.D. et al. PAWS1 controls cytoskeletal dynamics and cell migration through association with the SH3 adaptor CD2AP. J Cell Sci 131, jcs202390 (2018).

48. Fife, C., McCarroll, J. \& Kavallaris, M. Movers and shakers: cell cytoskeleton in cancer metastasis. British journal of pharmacology 171, 5507-5523 (2014).

49. Theriot, J.A. \& Mitchison, T.J. Actin microfilament dynamics in locomoting cells. Nature 352, 126 (1991).

50. Jayatilaka, H. et al. EB1 and cytoplasmic dynein mediate protrusion dynamics for efficient 3-dimensional cell migration. The FASEB Journal 32, 1207-1221 (2017).

51. Artym, V.V., Matsumoto, K., Mueller, S.C. \& Yamada, K.M. Dynamic membrane remodeling at invadopodia differentiates invadopodia from podosomes. European journal of cell biology 90, 172-180 (2011).

52. Krause, M. \& Gautreau, A. Steering cell migration: lamellipodium dynamics and the regulation of directional persistence. Nature reviews Molecular cell biology 15, 577 (2014).

53. Yamaguchi, H., Wyckoff, J. \& Condeelis, J. Cell migration in tumors. Current opinion in cell biology 17, 559-564 (2005).

54. Charras, G. \& Paluch, E. Blebs lead the way: how to migrate without lamellipodia. Nature reviews Molecular cell biology 9, 730 (2008).

55. Hausser, J. \& Alon, U. Tumour heterogeneity and the evolutionary trade-offs of cancer. Nature Reviews Cancer 20, 247-257 (2020).

56. Dagogo-Jack, I. \& Shaw, A.T. Tumour heterogeneity and resistance to cancer therapies. Nature reviews Clinical oncology 15, 81 (2018). 
1 57. Gandalovičová, A., Rosel, D. \& Brábek, J. in Approaching Complex Diseases 203-211 2 (Springer, 2020).

3 58. Fischer, R.S. et al. Contractility, focal adhesion orientation, and stress fiber

59. Kim, J. et al. The mechanics and dynamics of cancer cells sensing noisy 3D contact guidance. Proceedings of the National Academy of Sciences 118 (2021).

60. Brückner, D.B. et al. Learning the dynamics of cell-cell interactions in confined cell migration. Proceedings of the National Academy of Sciences 118 (2021).

61. Chrisafis, G. et al. Collective cancer cell invasion requires RNA accumulation at the invasive front. Proceedings of the National Academy of Sciences 117, 2742327434 (2020).

62. Guo, Z. et al. Validation of a vasculogenesis microfluidic model for radiobiological studies of the human microvasculature. Advanced Materials Technologies 4, 1800726 (2019). 


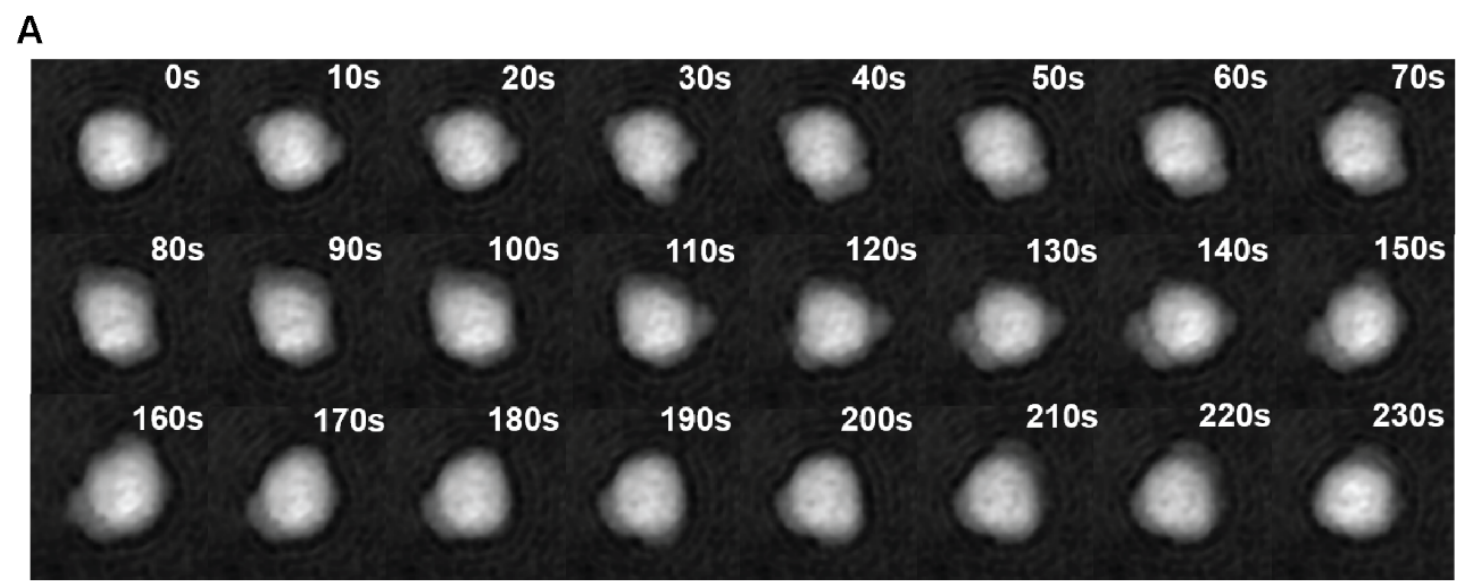

B

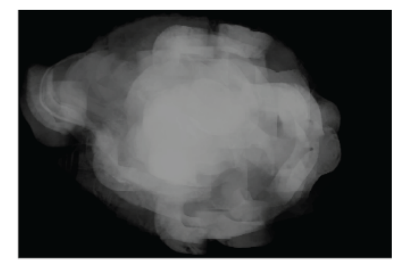

E

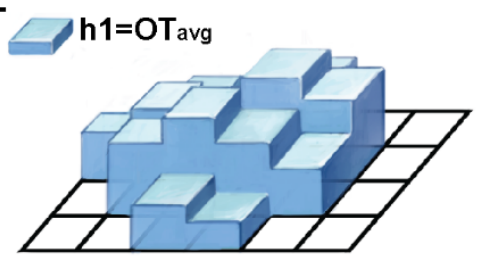

H

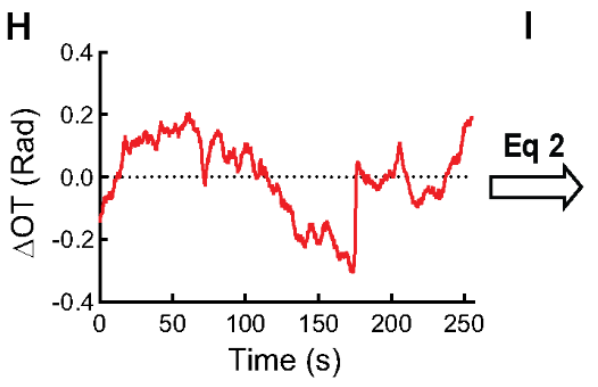

C

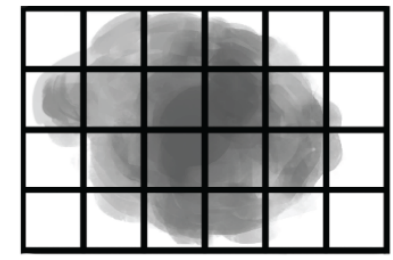

F
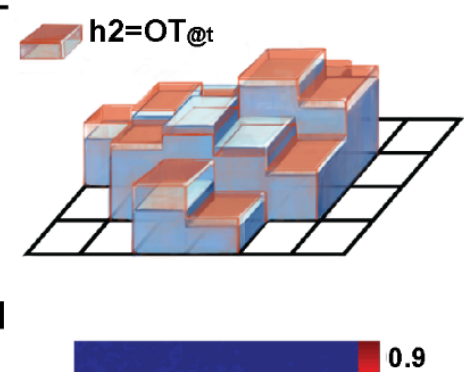

0.9

D

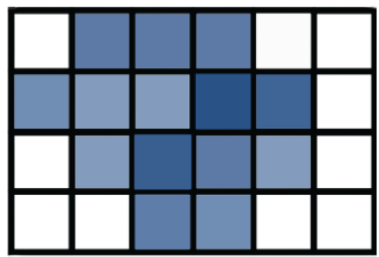

G

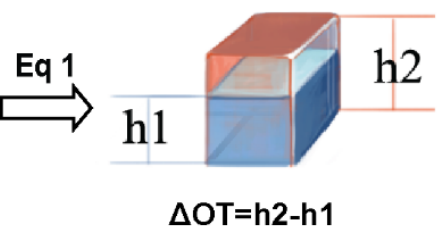

J

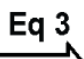

Micromotion

Index of the

Single Cell

0.293

3 Figure 1 Probing and quantifying optical cellular micromotion in 3D environments. (A)

4 Time sequence image series of single cell in fibrin gel imaged with DHM. (B-J) Schematic

5 illustration of the work flow for single cell micromotion measurement. (B) DHM imaging of a

6 single cell (C) Segmentation of cell from background. (D) Pixel based histogram plotting of mean

7 optical thickness (OT) over entire image period. (E) Pixel based histogram of mean OT over 
1 entire image period. (F) OT at specific time point $\mathrm{T}_{\mathrm{a} \cdot}(\mathbf{G})$ Calculation of $\Delta \mathrm{OT}\left(\mathrm{OT} @ \mathrm{~T}_{\mathrm{a}}-\mathrm{Mean}\right.$ OT)

2 using Eq1. (H) Plot of $\Delta \mathrm{OT}$ fluctuation over time. (I) Calculated mean square of fluctuation

3 (micromotion index) using Eq 2 and mapping over entire cell. (J) Averaging micromotion index

4 of all pixels in the cell.

A

Live 3D Fixed 3D Live 2D Fixed 2D

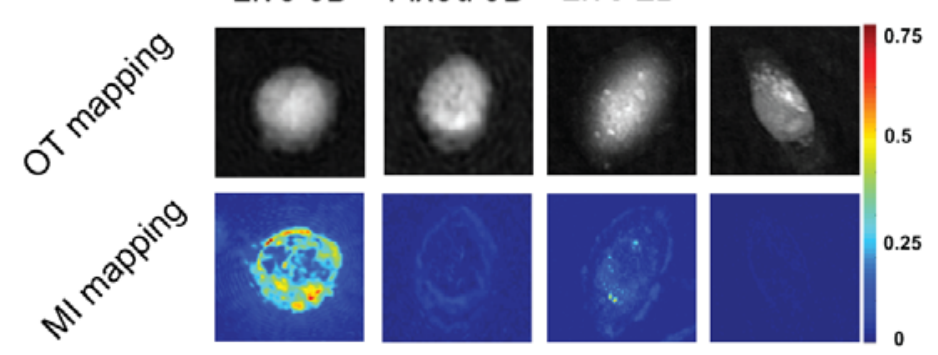

B
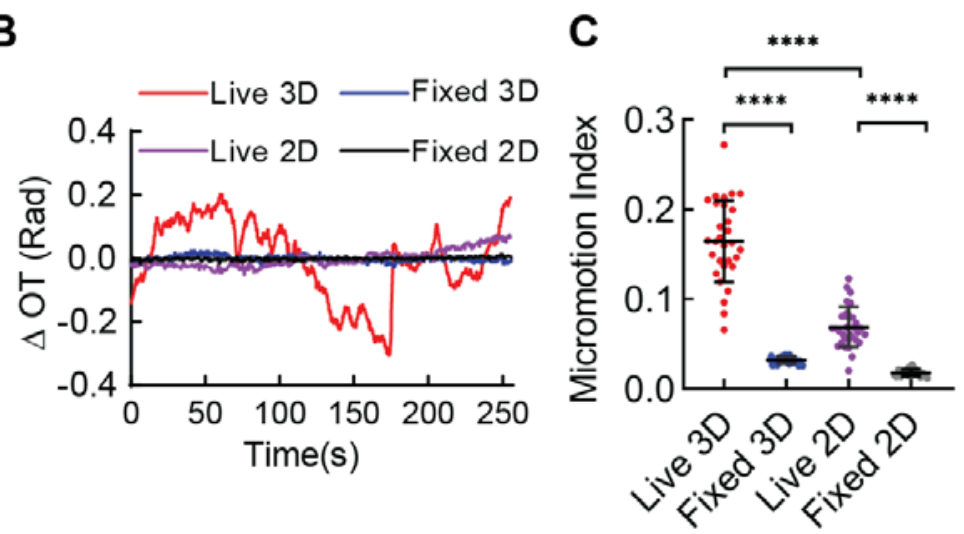

7 Figure 2 Measured 3D cellular micromotions are mainly associated with live cell behaviors

8 and significantly higher than in 2D. (A) Fluctuation curves of live and fixed MDA-MB-231

9 cells in 2D and 3D (at 24h). (B) OT and MI mapping of live and fixed cells in 3D and 2D. (C) MI

10 measurements for live and fixed cells in 3D and 2D (one point represents the MI of a single cell).

11 Error bars are SD (****, $\mathrm{P}<0.0001$ unpaired two-sided t-test) 


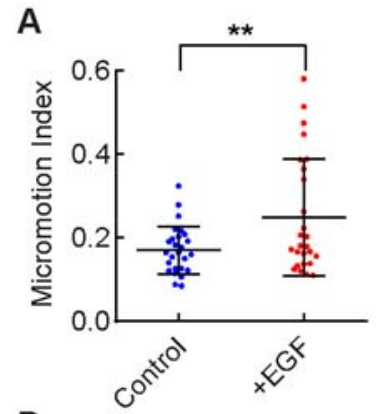

D
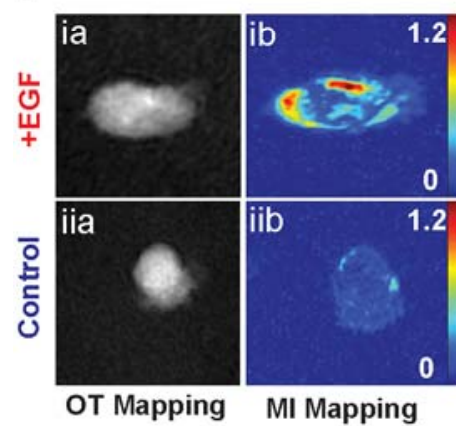

B
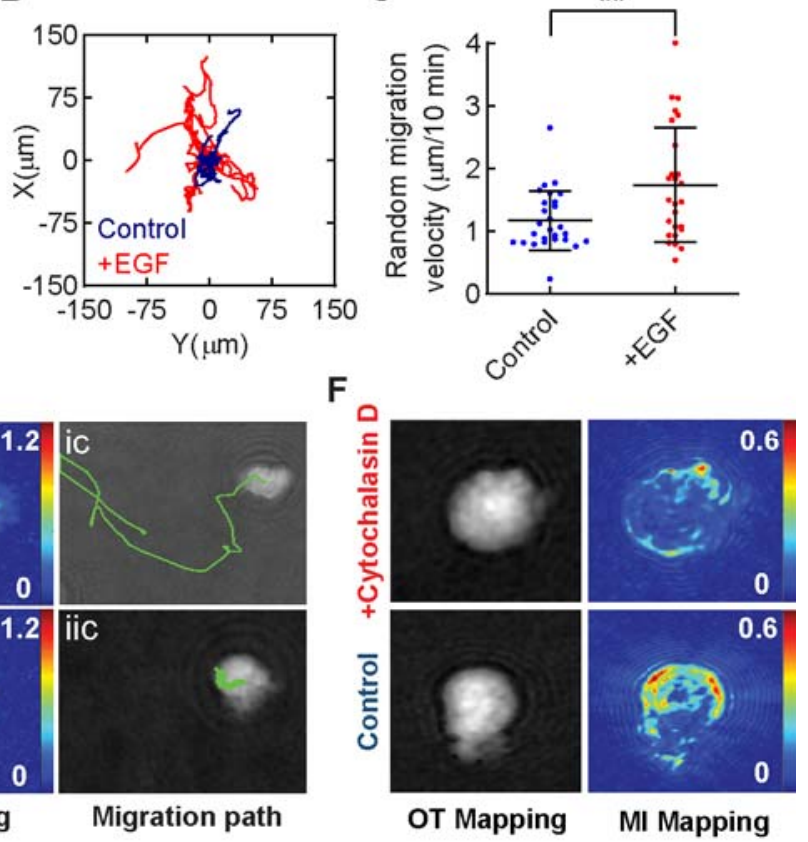

$E$

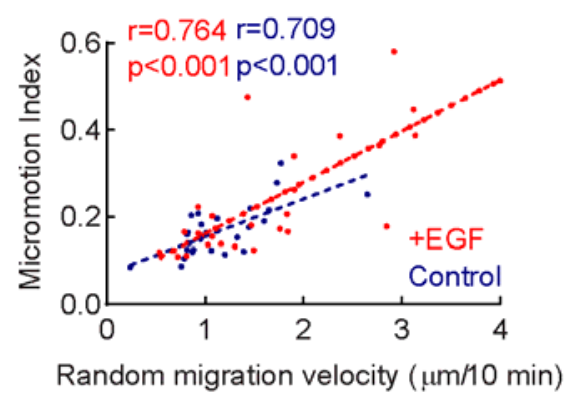

F

C
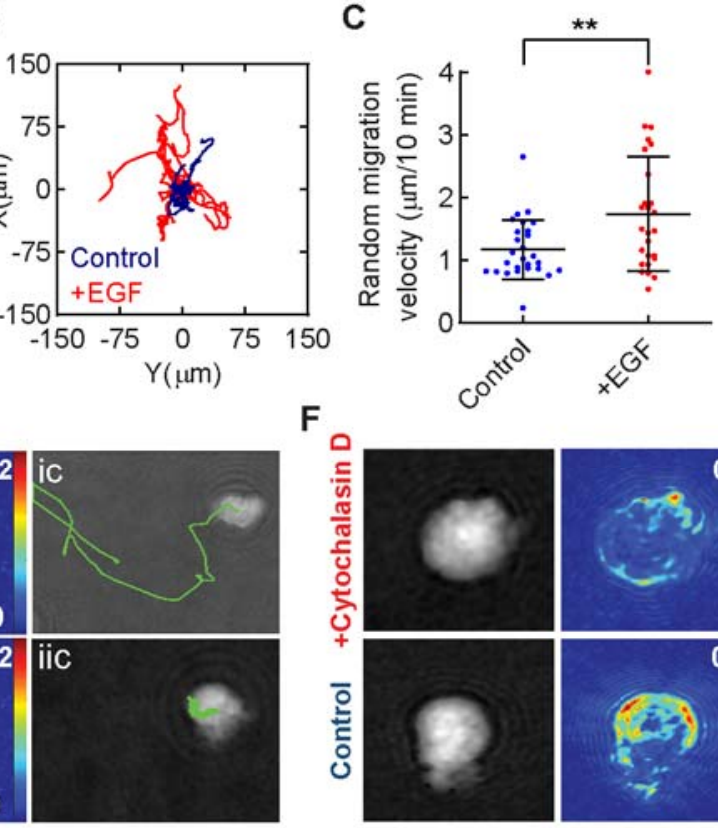

Migration path

G

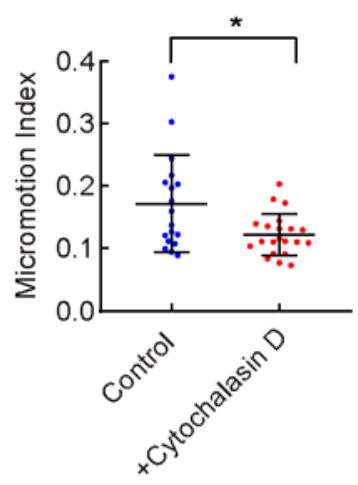

2 Figure 3 Micromotion and motility in fibrin gel are correlated at the single cell level.

3 (A) MIs measured for MDA-MB-231 cells treated or not with EGF. (B) Typical migration paths 4 recorded over $12 \mathrm{~h}$ for two single cells within fibrin gel supplemented or not with EGF. (C) Mean 5 migration speeds in fibrin gel calculated for single cells treated or not with EGF (2D projection). (D) OT mapping, MI mapping and migration tracking of a typical cell in the +EGF (Dia-Dic) and control (Diia-Diic) groups. (E) Scatter plot of the MI vs mean migration speed for single cells in

8 +EGF (red) and control (blue) groups. (F) OT mapping and MI mapping of a typical cell in the

$9+$ Cytochalasin D and control groups. (G) MIs measured of cells treated or not with Cytochalasin D. Error bars are SDs $(* \mathrm{P}<0.05, * * \mathrm{P}<0.01$, unpaired two-sided t-test $)$ 

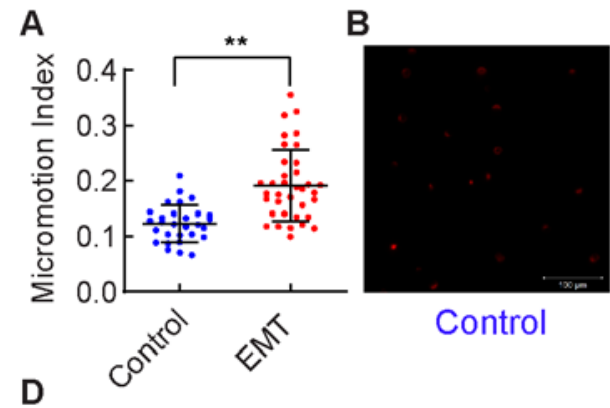

Control

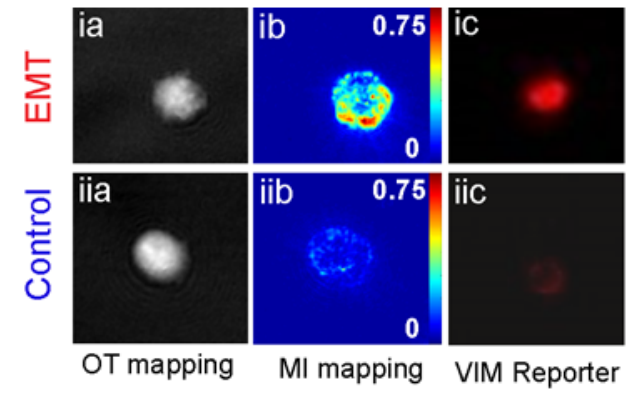

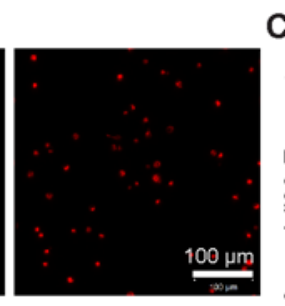

EMT

E
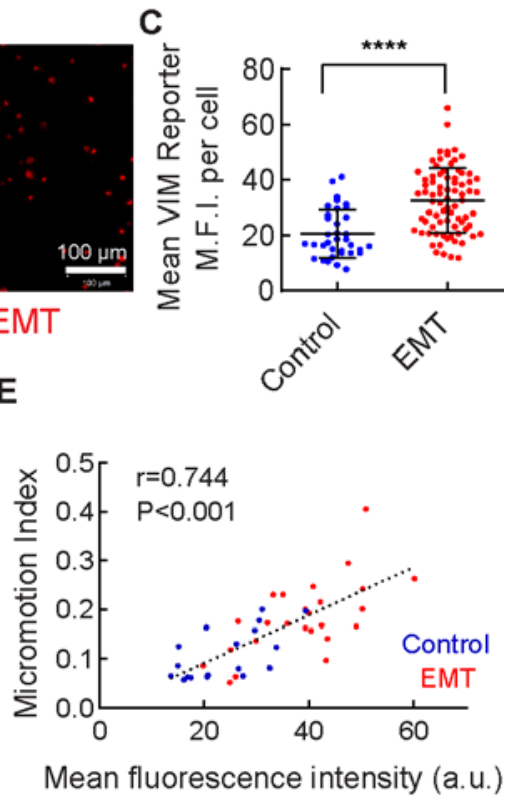

2 Figure 4 Acquisition of EMT phenotype results in increase in cellular MI. (A) MIs for EMT

3 and control groups of A549 VIM RFP cells. (B) Confocal imaging of A549 VIM-RFP cells

4 within fibrin gel with and without EMT induction. Scale bar: $100 \mu \mathrm{m}$. (C) MFIs for EMT and

5 control A549 VIM RFP cells (D) Representative OT mapping, MI mapping and RFP imaging for

6 A549 VIM-RFP cells with (D-i) or without (D-ii) EMT treatment. (E) Scatter plot of the MIs vs

7 mean RFP fluorescence intensity for single cells in the EMT (red) and control (blue) groups.

8 Error bars are $\mathrm{SD}(* * *, \mathrm{P}<0.001$ unpaired two-sided $\mathrm{t}$-test). 

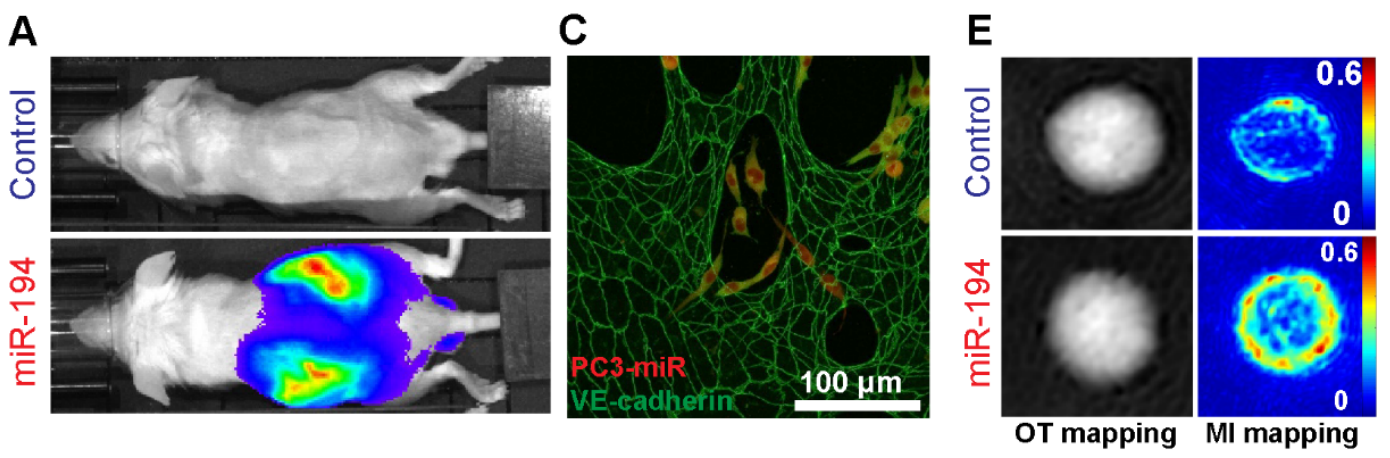

B

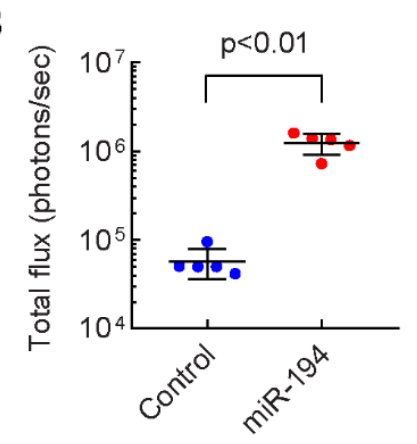

D
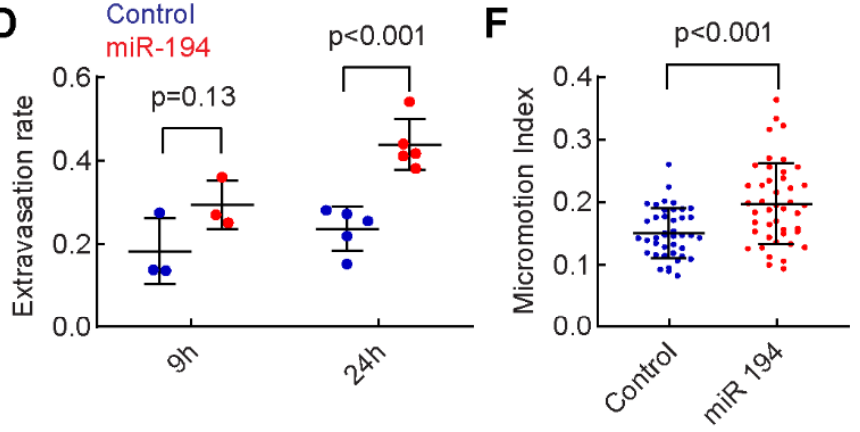

G

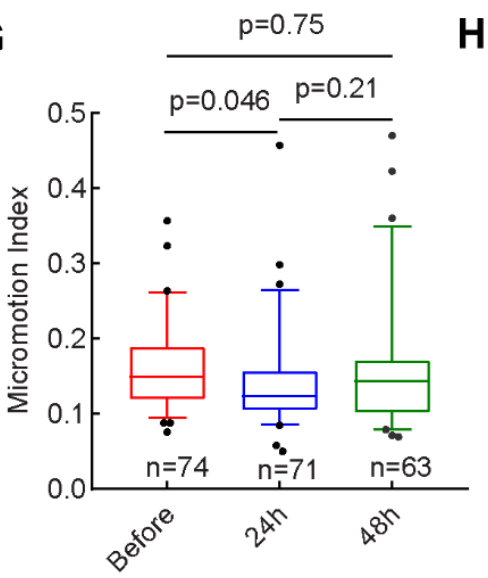

GNST
H
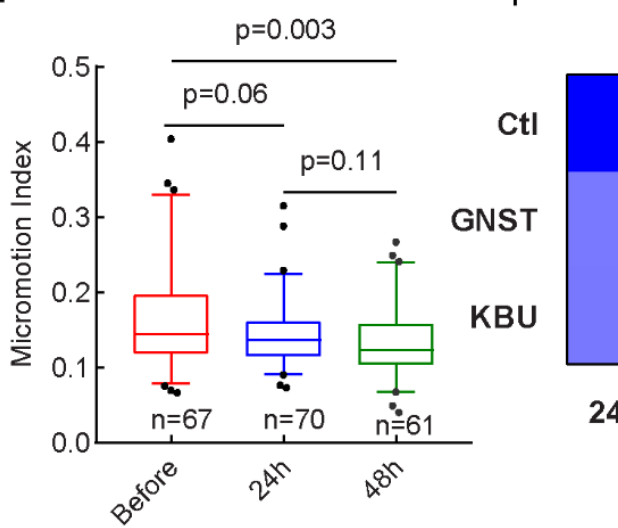

1.0

0.9

0.8

2 Figure 5 Highly metastatic cells that display higher optical cellular micromotion can be

3 reduced by migrastatic drugs. (A) Images showing whole-animal bioluminescent imaging of

4 representative mice in each group at 6 weeks after mice were injected with control cells or miR-

5 194-overexpressing cells. (B) Luciferase intensity as assessed by whole-animal bioluminescent

6 imaging ( $\mathrm{n}=5$ in each group, one data point represents a single mouse). (C) Confocal imaging of

7 PC-3 cells extravasation in a microfluidic microvasculature model. Vasculature is stained for VE-

8 cadherin (green) and PC-3 are labelled with CellTracker ${ }^{\mathrm{TM}}$ CMTPX dye (red). (D) PC-3 cells

9 extravasation ratios in the microfluidic microvasculature model for non-transfected control 
1 (black) and miR-194 overexpressing cells (red) at 9h and 24 after perfusion. One point represents 2 a separate microfluidic device. (E) Typical images of PC-3 cells OT and MI mapping. (F) MIs for 3 miR-194 overexpressing PC-3 cells and control group. (G-H) MIs of PC-3 before, 24h and 48h 4 after being treated with $10 \mu \mathrm{M}$ of genistein or KBU 2046. Error bars are SD (unpaired two-sided 5 t-test). (I) Fold changes of mean MIs of PC-3 cells at different time points after being treated with 6 genistein or KBU 2046, or without treatment, compared to before treatment (set before treatment 7 as 1$)$. 
A

\begin{tabular}{|c|c|c|c|c|c|c|}
\hline $0 s$ & 105 & $20 s$ & $30 s$ & $40 s$ & $50 s$ & 605 \\
\hline $80 s$ & $90 \mathrm{~s}$ & $100 s$ & $110 \mathrm{~s}$ & $120 s$ & $130 s$ & $140 \mathrm{~s}$ \\
\hline $160 \mathrm{~s}$ & $170 \mathrm{~s}$ & $180 s$ & $190 s$ & 2005 & $210 s$ & $220 \mathrm{~s}$ \\
\hline
\end{tabular}

B

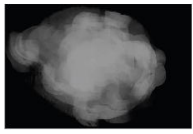

E

E h1=OTavg

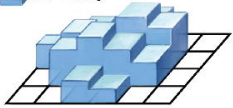

H

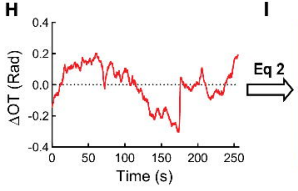

F
D

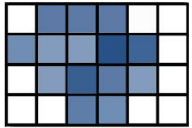

G

$\mathrm{C}^{\mathrm{h} 2=\mathrm{OT}}$

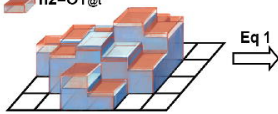

h1

$\Delta \mathrm{OT}=\mathrm{h} 1-\mathrm{h} 2$

J

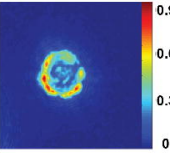

$\mathrm{h} 2$

Micromotion Index of the Single Cell

0.293 
Live 3D Fixed 3D Live 2D Fixed 2D
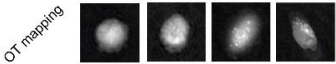

6.75
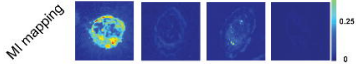

B
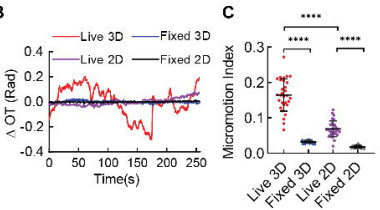
A

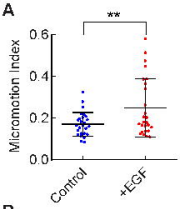

D

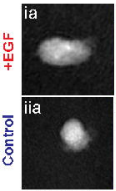

OT Mapping MI Mapping

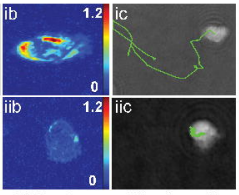

Migration path

B $\quad$ C

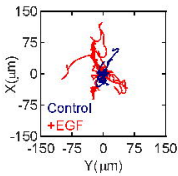

F

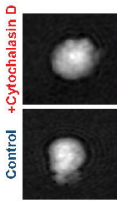

OT Mapping
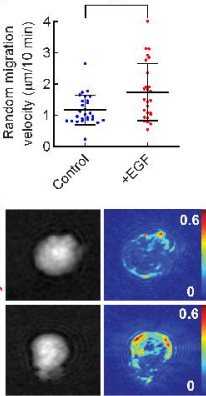

MI Mapping

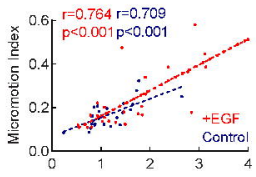

6

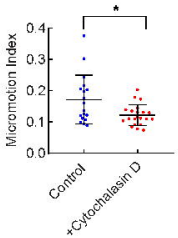


A

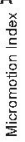

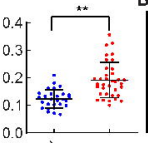

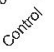

D

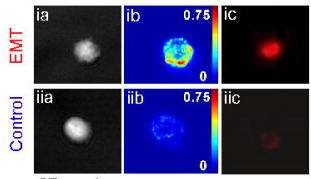

OT mapping

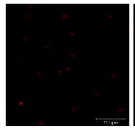

Control

EMT

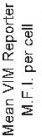

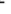

たなな丈

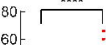

$100 \mathrm{\mu m}$

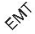

E

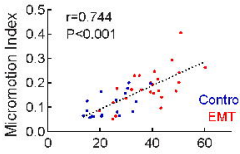

Mean fluorescence intensity (a.u.) 
A

흘

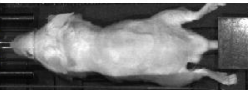

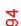

产

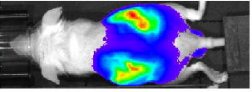

B

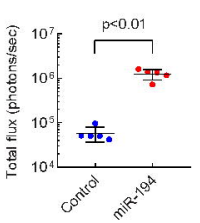

G

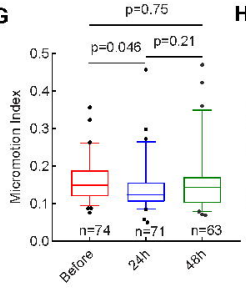

GNST

H
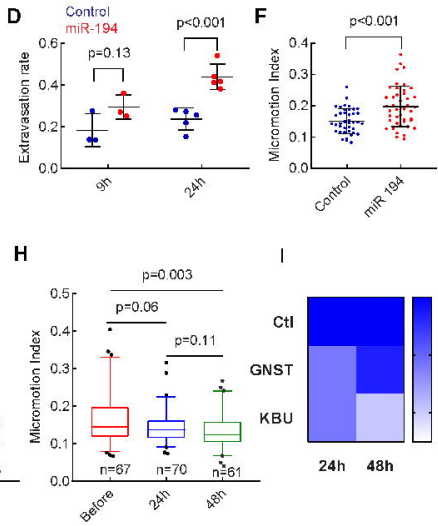

KBU2046

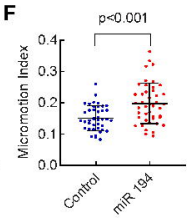

E
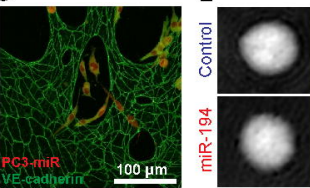

0.6

$\frac{5}{\frac{5}{5}}$

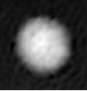

OT mapping

0

MI mapping

0

0.6

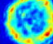

0$$
\text { . }
$$

1.0

0.9

0.8 